\title{
Inhalational anthrax
}

INSERM

\section{Source}

INSERM. (1999). Orphanet: an online rare disease and orphan drug data base.

Inhalational anthrax. ORPHA:247257

Inhalational anthrax is a rare acute systemic infection caused by the inhalation of Bacillus anthracis spores (e.g. through infected animal products, bioterrorism) and characterized by an initial stage where patients present with non specific symptoms (fever, cough, chills, fatigue) that is followed by an acute phase during which hemorrhagic mediastinitis occurs that can progress into meningitis, gastrointestinal involvement, and refractory shock, that can be fatal, if left untreated. 